\title{
Diagramas para el manejo de la densidad en bosques mixtos e irregulares de Durango, México
}

\author{
Density management diagrams in mixed and uneven-aged forests of Durango, México
}

\author{
Sacramento Corral-Rivas a, Juan G Álvarez-González b , José J Corral-Rivas c \\ Christian Wehenkel ${ }^{\mathrm{c}}$, Carlos A López-Sánchez ${ }^{\mathrm{c} *}$
*Autor de correspondencia: c Universidad Juárez del Estado de Durango, Instituto de Silvicultura e Industria de la Madera, Carretera Mazatlán, Km 5.5, 34120, Durango, México, tel.: +52618 8251886, calopez@ujed.mx
a Instituto Tecnológico de El Salto, Mesa del Tecnológico, 34942 El Salto P.N., Durango, México.
${ }^{\mathrm{b}}$ Universidad de Santiago de Compostela, Escuela Politécnica Superior, Departamento de Ingeniería Agroforestal, R/ Benigno Ledo, Campus Universitario, 27002, Lugo, España.

\begin{abstract}
SUMMARY
Density management diagrams (DMD) are graphical tools used in the design of silvicultural regimes for both even-aged and unevenaged forests. They depict the relationship among stand density, average tree size, stand yield and dominant height, based upon relevant ecological and allometric relationships such as the self-thinning rule, the yield-density effect, and site index curves. DMD effectively summarize stand structural descriptors, and are therefore helpful in determining stand characteristics needed to achieve a range of management goals. In this work several DMD were built for conifer and broadleaf species in two conditions of productivity in mixed and uneven-aged forests in Northwest of Durango, Mexico. Additionally, two DMD were developed including all species present in the stand (conifer + broadleaves) for each level of productivity and finally a DMD as the sum of the two levels of productivity for all species into stand was developed. We used 15,360 temporal sample plots from a region-wide forest inventory. To determine the maximum density isoline we selected 333 plots with maximum density (considering the group of species belonging to conifers). Site index curves were used to assess the time taken by stands to progress along their development trajectories. The DMD of species grouped into conifers in high productivity level was used as a case study to illustrate how the diagram can be used to assess current functionality of the forest, forecast its likely development and compare alternative management strategies.
\end{abstract}

Key words: maximum density, relative spacing index, simultaneous fitting.

\section{RESUMEN}

Los diagramas para el manejo de la densidad (DMD) son herramientas gráficas utilizadas en el diseño de los regímenes silvícolas de bosques regulares e irregulares; representando las relaciones entre la densidad, el diámetro cuadrático, el incremento y la altura dominante del rodal mediante relaciones alométricas y ecológicas como: regla del autoaclareo, efecto de la densidad sobre el crecimiento y calidad de sitio. Los DMD describen eficientemente la estructura del rodal, siendo útiles en la determinación de las características de la producción final. Se construyeron DMD, para coníferas y latifoliadas, en dos condiciones de productividad en bosques mixtos e irregulares del noroeste de Durango, México. Adicionalmente, se construyeron dos DMD que incluyen todas las especies presentes en el rodal para cada nivel de productividad y un DMD elaborado como la suma de los dos niveles de productividad de todas las especies. Los datos empleados provienen de 15.360 sitios temporales de muestreo, de los cuales se seleccionaron 333 que representan los valores máximos de densidad (para el grupo de especies de coníferas) para representar la isolínea superior de los DMD. El DMD de las especies de coníferas en su nivel de productividad alto fue empleado como un caso de estudio para ilustrar como estos diagramas pueden ser usados en evaluar las condiciones actuales del rodal, pronosticar su desarrollo y comparar diferentes alternativas de manejo.

Palabras clave: límite de máxima densidad, índice de espaciamiento relativo, ajuste simultáneo.

\section{INTRODUCCIÓN}

El manejo de la densidad del rodal consiste en el control del espacio disponible para el crecimiento de los árboles por medio de cortas intermedias para lograr diferentes objetivos de manejo (Newton 1997). La determinación de los niveles adecuados de densidad para una masa forestal en una situación determinada es un proceso complejo que depende de factores biológicos, tecnológicos, económicos y operacionales (Dean y Baldwin 1996).

Desde el punto de vista de producción maderable, las alternativas para el manejo de la densidad que se pueden plantear para las masas forestales en una situación determinada consisten en aprovechar al máximo los recursos 
de la calidad de estación (Barrio-Anta y Álvarez-González 2005). Sin embargo, este máximo aprovechamiento se puede lograr con un abanico razonable de espesuras que contemple los límites superior e inferior que no conviene sobrepasar. El límite superior correspondería con la máxima densidad que es capaz de sustentar el sitio de modo que todos sus recursos sean aprovechados por el rodal aunque cada árbol sólo podría obtener lo imprescindible para sobrevivir, esta situación corresponde con la definición clásica de monte normal (Dean y Baldwin 1996). Puesto que los recursos del medio (espacio físico, agua, nutrientes y luz) necesarios para el desarrollo de una masa forestal son limitados, cuando en una masa se supera este límite, algunos árboles deben morir para posibilitar la existencia de recursos adicionales disponibles que permitan el crecimiento de los restantes individuos, por lo tanto, se inicia el autoaclareo o mortalidad natural, que debe ser anticipado mediante cortas intermedias, denominadas aclareos (Dean y Baldwin 1996). El límite inferior se establece cuando todos los recursos del sitio son utilizados por la masa de modo que cada uno de los árboles que la constituye asimila todo lo que su condición genética y edad le permite, es decir, desarrolla su máxima capacidad de crecimiento. Al mantener la masa por debajo del límite inferior se pierde capacidad productiva y, por tanto, se infrautiliza el sitio. Aunque los ensayos de campo son la mejor forma de determinar los límites teóricos antes mencionados y la edad adecuada para realizar las cortas intermedias, estos tienen dos serias limitaciones (Dean y Baldwin 1993): (i) se necesitan muchos años para completarse y, (ii) los resultados no pueden ser aplicados con fiabilidad cuando la calidad de estación o los objetivos de manejo son distintos a los de los ensayos. Un paliativo que se puede conseguir en poco tiempo es un diagrama para el manejo de la densidad (DMD), que representa ciertas condiciones promedio del rodal, y gráficamente muestra la relación entre rendimiento, densidad y mortalidad para las diferentes etapas de desarrollo de la masa (Newton y Weetman 1994).

El uso de los DMD es uno de los métodos más efectivos para el diseño, control y evolución de la densidad del rodal bajo diferentes regímenes de manejo. Su uso fue inicialmente desarrollado por científicos japoneses a principios de 1960 (Tadaki 1963), posteriormente en los años 70 y 80 se realizaron varias modificaciones al concepto original de los DMD, incorporando teorías de la producción forestal (Newton 1997). Actualmente, estos diagramas han sido desarrollados para caracterizar las existencias maderables de las especies presentes en el rodal usando índices que relacionan el tamaño medio de los árboles (volumen, altura y diámetro) con la densidad (número de árboles por hectárea). Diversos índices de densidad han sido usados en el desarrollo de DMD para plantaciones forestales, destacando entre ellos: el índice de densidad del rodal propuesto por Reineke (1933); la regla del auto aclareo propuesta por Yoda et al. (1963), y el índice de espacio de crecimiento relativo de Drew y Flewelling (1979).
La mayor parte de los bosques del estado de Durango (México) corresponde a una mezcla de especies de los géneros Pinus y Quercus, a menudo mezclados con pies aislados de los géneros Pseudotsuga, Arbutus y Juniperus, entre otras especies. Estos rodales se caracterizan por la presencia de, al menos, dos estratos arbóreos verticales: uno superior formado por una mezcla de especies de pino y pies aislados de otras coníferas con diferentes clases de edad y, un segundo estrato por debajo del anterior formado fundamentalmente por una mezcla de latifoliadas, también pertenecientes a diferentes clases de edad [ver Wehenkel et al. (2011) para una descripción de la estructura de estos bosques]. Estos bosques son la primera reserva forestal del país, cubren 5,5 millones de hectáreas y aportan cerca de la cuarta parte de la producción de madera de México (SRNyMA 2006).

La gestión forestal de estos bosques requiere de herramientas cuantitativas, como los DMD, que permitan predecir los niveles de crecimiento y rendimiento maderable, controlar la densidad del rodal y programar las cortas. Sin embargo, a la fecha, en masas mixtas e irregulares del norte de México no se han desarrollado herramientas de esta índole, y los trabajos reportados se refieren a una sola especie y para un rango de edades y calidad de sitio muy específico (Aguirre et al. 1994, Márquez y Álvarez 1995). Así pues, la hipótesis es que la evolución de las masas mixtas e irregulares puede ser modelizada empleando la estructura de los diagramas de manejo de la densidad, por lo que el objetivo del presente trabajo es desarrollar DMD basados en el índice de espacio de crecimiento relativo para este tipo de masas, considerando dos niveles de productividad definidos por la altura dominante y la cobertura de copas de los rodales.

\section{MÉTODOS}

Área de estudio. El presente trabajo se llevó a cabo en una superficie de 26.000 hectáreas de bosque de clima templado frío dentro de los límites del Ejido San Diego de Tezains del estado de Durango, México. Geográficamente el área de estudio se delimita por las coordenadas $105^{\circ} 53^{\prime}$ $36^{\prime \prime}$ y $106^{\circ} 12^{\prime} 40^{\prime \prime}$ de longitud oeste y $24^{\circ} 48^{\prime} 16^{\prime \prime}$ y $25^{\circ}$ 13 ' 32" de latitud norte (figura 1). El tipo de vegetación predominante corresponde a bosques mixtos e irregulares de Pino - Encino. La altura sobre el nivel del mar varía desde los 1.400 a los 3.000 metros. El clima que prevalece corresponde a un clima templado semifrío con un régimen de precipitación anual que fluctúa de 800 a 1.100 $\mathrm{mm}$ y una temperatura media anual que varía de $8^{\circ} \mathrm{C}$ en las partes más altas hasta los $24^{\circ} \mathrm{C}$ en las partes más bajas (García 1981).

Datos. Los datos provinieron de 15.360 parcelas temporales de muestreo que fueron establecidas y medidas en el año 2009 para el inventario de manejo forestal maderable del ejido mediante muestreo aleatorio estratificado 


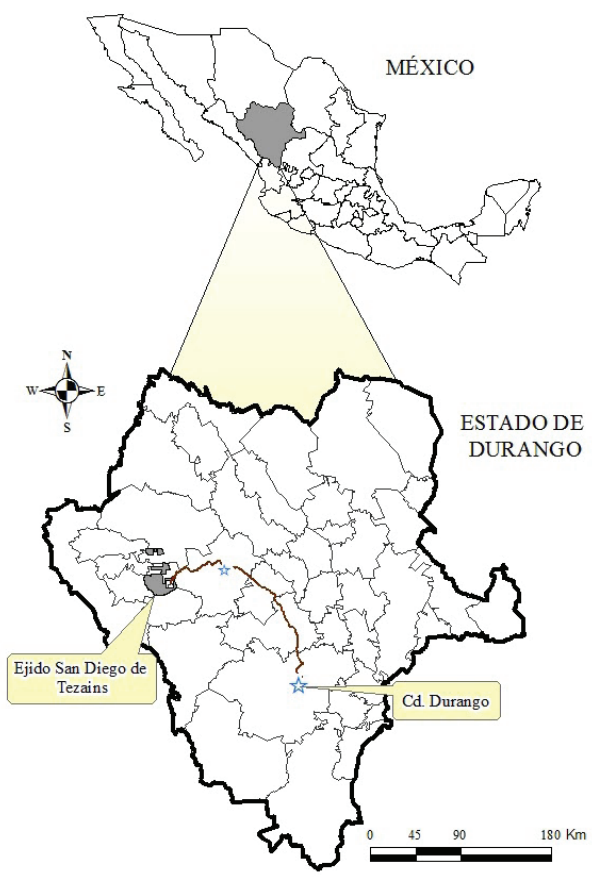

Figura 1. Localización del área de estudio.

Location of the study area. en 4.193 rodales; en su mayoría mixtos e irregulares. Los rodales fueron clasificados en dos niveles de productividad de la siguiente manera: (i) nivel alto, para aquellos rodales que tienen una cobertura de copas mayor al $50 \%$ y un índice de sitio correspondiente a una altura dominante del rodal mayor o igual a 16 metros a los 60 años (36,3\% de la superficie estudiada); y (ii) nivel medio, rodales que tienen una cobertura de copas que oscila de $20 \%$ a $50 \%$ y un índice de sitio correspondiente a una altura dominante menor a 16 metros a los 60 años $(31,7 \%$ de la superficie estudiada) (cuadro 1).

En este trabajo la altura dominante $\left(H_{0}\right)$ se estimó como el promedio de la altura de los 100 árboles más gruesos por hectárea (Assmann 1961) y la edad como el valor medio del conteo de anillos de tres árboles dominantes por rodal. Los DMD se desarrollaron para tres grupos de especies: (i) coníferas, que incluyó a todas aquellas especies que pertenecen a los géneros Pinus ( $P$. arizonica Engelm, $P$. ayacahuite Ehrenb, $P$. durangensis Martínez, $P$. herrerae Martínez, P lumholtzii Robins et Ferns, $P$. teocote Schlecht et Cham, P. douglasina Martínez), Pseudotsuga menziesii Mirb, Cupressus spp. y Juniperus spp.; (ii) latifoliadas, todas aquellas especies que pertenecen a los géneros Quercus (Q. candicans Née, Q. coccolobifolia Trel,

Cuadro 1. Estadísticos de las principales variables usadas en la construcción de los DMD para los grupos de especies y niveles de productividad considerados.

Statistics for the main variables used in the construction of the DMD for groups of species and productivity levels considered.

\begin{tabular}{|c|c|c|c|c|c|c|}
\hline \multirow{2}{*}{$\begin{array}{c}\text { Nivel de } \\
\text { productividad }\end{array}$} & Variable & $\mathrm{N}^{\mathrm{o}}$ rodales & Media & Desviación estándar & Mínimo & Máximo \\
\hline & \multicolumn{6}{|c|}{ Coníferas } \\
\hline \multirow{4}{*}{ Alto } & Altura dominante (m) & 1.432 & 17,4 & 3,3 & 9,4 & 29,3 \\
\hline & Diámetro cuadrático $(\mathrm{cm})$ & 1.432 & 25,5 & 4,5 & 15,8 & 44,7 \\
\hline & Densidad $\left(\mathrm{N} \mathrm{ha}^{-1}\right)$ & 1.432 & 412,2 & 165,0 & 50,0 & 1147,0 \\
\hline & Volumen $\left(\mathrm{m}^{3} \mathrm{ha}^{-1}\right)$ & 1.432 & 193,6 & 70,9 & 44,8 & 540,9 \\
\hline \multirow{5}{*}{ Medio } & Altura dominante (m) & 1.376 & 12,6 & 2,4 & 6,8 & 22,1 \\
\hline & Diámetro cuadrático $(\mathrm{cm})$ & 1.376 & 24,5 & 4,0 & 14,7 & 43,6 \\
\hline & Densidad $\left(\mathrm{N} \mathrm{ha}^{-1}\right)$ & 1.376 & 307,0 & 111,5 & 55,0 & 815,0 \\
\hline & Volumen $\left(\mathrm{m}^{3} \mathrm{ha}^{-1}\right)$ & 1.376 & 130,0 & 40,3 & 41,5 & 291,0 \\
\hline & \multicolumn{6}{|c|}{ Latifoliadas } \\
\hline \multirow{4}{*}{ Alto } & Altura dominante (m) & 1.430 & 9,4 & 2,5 & 3,8 & 19,4 \\
\hline & Diámetro cuadrático $(\mathrm{cm})$ & 1.430 & 27,6 & 7,3 & 11,6 & 65,0 \\
\hline & Densidad $\left(\mathrm{N} \mathrm{ha}^{-1}\right)$ & 1.430 & 205,1 & 130,4 & 6,0 & 1065,0 \\
\hline & Volumen $\left(\mathrm{m}^{3} \mathrm{ha}^{-1}\right)$ & 1.430 & 76,9 & 50,1 & 0,3 & 465,6 \\
\hline \multirow{4}{*}{ Medio } & Altura dominante (m) & 1.375 & 8,9 & 2,3 & 3,9 & 19,0 \\
\hline & Diámetro cuadrático $(\mathrm{cm})$ & 1.375 & 26,0 & 6,4 & 10,0 & 59,4 \\
\hline & Densidad $\left(\mathrm{N} \mathrm{ha}^{-1}\right)$ & 1.375 & 132,7 & 73,6 & 3,0 & 740,0 \\
\hline & Volumen $\left(\mathrm{m}^{3} \mathrm{ha}^{-1}\right)$ & 1.375 & 41,6 & 22,0 & 0,1 & 144,5 \\
\hline \multicolumn{7}{|c|}{ Coníferas + Latifoliadas (total) } \\
\hline Alto & Volumen $\left(\mathrm{m}^{3} \mathrm{ha}^{-1}\right)$ & 1.432 & 290,7 & 90,1 & 120,2 & 869,8 \\
\hline Medio & Volumen $\left(\mathrm{m}^{3} \mathrm{ha}^{-1}\right)$ & 1.375 & 181,7 & 44,8 & 78,4 & 387,1 \\
\hline
\end{tabular}


Q. conzattii Trel, Q. cordifolia Trel, Q. crassifolia Humb et Bonpl, Q. durifolia Seemen ex Loes, Q. fulva Liebm, Q. grisea Liebm, Q. microphylla Née, Q. obtusata Bonpl, $Q$. resinosa Liebm, Q. rugosa Née, Q. rysophylla Weath, Q. sideroxyla Humb. et Bonpl, Q. urbanii Trel), Arbutus xalapensis Kunth y Alnus spp.; (iii) coníferas y latifoliadas, incluyendo a todas las especies registradas en los sitios de inventario.

El índice de Hart-Becking (Wilson 1946), o también conocido como índice de espacio de crecimiento relativo $(R S)$, fue empleado en este trabajo para la construcción de los DMD. Una ventaja de este índice es que puede utilizarse en el desarrollo de DMD independientes de la edad del rodal (Long 1985) y puede ser adaptado para bosques regulares e irregulares (Gadow y Hui 1999); representando por tanto, una buena herramienta silvícola para el manejo de las masas mixtas e irregulares que caracterizan los bosques del noroeste de México.

Los DMD se construyeron gráficamente a partir de dos ejes de coordenadas que corresponden con la altura dominante del rodal (eje X) y el número de árboles por hectárea (eje Y), y sobre los que se representan isolíneas de los valores del índice de espacio de crecimiento relativo (ecuación [1]) e isolíneas de las variables dependientes de las relaciones alométricas: (i) relaciona el diámetro medio cuadrático con el número de árboles por hectárea y la altura dominante del rodal (ecuación [2]) y; (ii) relaciona el volumen del rodal con el número de árboles por hectárea, el diámetro medio cuadrático y la altura dominante del rodal como un indicador de la calidad de estación (ecuación [3]). Estas relaciones alométricas tienen consistencia silvícola y biológica; el valor del diámetro medio cuadrático va a estar condicionado por los tratamientos silvícolas realizados (que determinan el número de árboles por hectárea) y por la calidad de estación (caracterizada por la altura dominante); por otro lado, el valor del volumen del rodal está directamente relacionado con el área basal de la masa (cuyo valor depende del número de árboles por hectárea y del diámetro medio cuadrático) y con la altura dominante del rodal. Las expresiones de las ecuaciones empleadas en la construcción de los DMD son las siguientes:

$$
\begin{gathered}
R S \%=\frac{\sqrt{\frac{20000}{N \sqrt{3}}}}{H_{0}} 100 \Rightarrow N=\left[\frac{2 \cdot 10^{8}}{\sqrt{3} H_{0}^{2} R S^{2}}\right] \\
d_{g}=\beta_{1} N^{\beta_{2}} H_{0}^{\beta_{3}} \Rightarrow N=\left[\frac{d_{g}}{\beta_{1} H_{0}^{\beta_{3}}}\right]^{1 / \beta_{2}} \\
V=\beta_{4} d_{g}^{\beta_{5}} N^{\beta_{6}} H_{0}^{\beta_{7}} \Rightarrow N=\left[\frac{V}{\beta_{4} \beta_{1}^{\beta_{5}} H_{0}^{\beta_{3} \beta_{5}+\beta_{7}}}\right]^{1 / \beta_{2} \beta_{5}+\beta_{6}}
\end{gathered}
$$

Donde, $R S=$ índice de espacio de crecimiento relativo de Hart-Becking $(\%) ; d_{\mathrm{g}}=$ diámetro medio cuadrático $(\mathrm{cm})$;
$N=$ densidad del rodal (número de árboles ha-1); $H_{0}=$ altura dominante $(\mathrm{m}) ; V=$ volumen del rodal $\left(\mathrm{m}^{3} \mathrm{ha}^{-1}\right)$ y $\beta_{i}(i=1-7)$ $=$ coeficientes a ser estimados mediante técnicas de regresión.

El índice $R S$ se obtiene como el cociente de la distancia media entre árboles y la altura dominante. En este caso, para su cálculo se empleó la distancia media entre árboles correspondiente a un rodal con una distribución de los árboles al tresbolillo, ya que se adecúa más al estado de las masas estudiadas. La utilización de este índice para caracterizar los niveles de existencias maderables en los DMD desarrollados en este trabajo se justifica por diferentes razones, entre las cuales destacan las siguientes: (i) es independiente de la edad del rodal excepto para rodales muy jóvenes (Schütz 1990); (ii) desde el punto de vista biológico la evolución de la altura dominante del rodal puede utilizarse para establecer el intervalo de tiempo entre las cortas sucesivas (Duplat et al. 1996); y (iii) la relación dendrométrica que se establece en estos diagramas entre el crecimiento en altura dominante y la producción del rodal representa una herramienta silvícola para propósitos de manejo forestal, especialmente porque la planeación debe de hacerse a este nivel. Barrio-Anta y Álvarez (2005) desarrollaron DMD para establecer regímenes de corta para Quercus robur L. usando el índice Hart-Becking; LópezSánchez y Rodríguez-Soalleiro (2009) construyeron DMD basados en el índice Hart-Becking para representar las isolíneas del volumen y del riesgo de incendio de copas para Pseudotsuga menziesii; Pérez-Cruzado et al. (2011), construyeron DMD basados en el índice Hart-Becking para representar las isolíneas del volumen y biomasa aérea de masas de Pinus pinaster Ait., Eucalyptus nitens Deane et Maiden y Eucalyptus globulus Labill.

Teniendo en cuenta las ecuaciones [1], [2] y [3], despejando en cada una de ellas la densidad del rodal $(N)$ y fijando distintos valores de índice de Hart-Becking $(R S)$, diámetro medio cuadrático $\left(d_{\mathrm{g}}\right) \mathrm{y}$ volumen $(V)$, respectivamente (dentro del rango observado en la construcción del diagrama) se pueden obtener las expresiones que permiten calcular las isolíneas del $\mathrm{RS}, d_{\mathrm{g}} \mathrm{y} V$. Posteriormente, estas trayectorias se sobreponen en un diagrama cartesiano con la altura dominante $\left(H_{0}\right)$ en el eje de las abscisas y la densidad $(N)$ en el eje de las ordenadas con lo cual queda definido el DMD.

Dado que el sistema alométrico definido por las ecuaciones [2] y [3] tiene como variable instrumental al diámetro medio cuadrático $\left(d_{\mathrm{g}}\right)$, es decir, $d_{\mathrm{g}}$ es la variable dependiente de la ecuación [2], mientras que en la ecuación [3] es una variable independiente, fue necesario realizar el ajuste de ambas ecuaciones de manera simultánea para evitar la posible correlación de errores. Los ajustes se realizaron por nivel de productividad para los grupo de coníferas, latifoliadas, y para el total de las especies (coníferas + latifoliadas). Además, se desarrolló un DMD general, sin clasificar los rodales por clase de productividad. Por tanto, se construyeron un total de siete DMD, y la selección entre 
uno u otro en su uso práctico va a depender de cuál sea el grupo de especies prioritario en la toma de decisiones y del nivel de productividad del rodal.

En el caso de los diagramas construidos para todas las especies conjuntamente, la ecuación [3] se sustituyó por otra que estimara el volumen total del rodal como la suma de los volúmenes de los grupos de coníferas y latifoliadas (ecuación [4]).

$V t=\beta_{4} \beta_{1}^{\beta_{5}} N^{\beta_{2} \beta_{5}+\beta_{6}} H_{0}^{\beta_{3} \beta_{5}+\beta_{7}}+\beta_{8} \beta_{1}^{\beta_{9}} N^{\beta_{2} \beta_{9}+\beta_{10}} H_{0}^{\beta_{3} \beta_{9}+\beta_{11}}$

Donde, $\boldsymbol{\beta}_{1}, \ldots, \boldsymbol{\beta}_{11}=$ parámetros obtenidos en el ajuste.

Dada la especial estructura de la ecuación [4], no es posible despejar el valor de la densidad $(N)$ para representar gráficamente las isolíneas de volumen en el diagrama, por lo que el valor de $N$ para cada combinación de altura dominante y densidad fue resuelto mediante un proceso iterativo.

Las variables $N$ y $H_{0}$ usadas en el ajuste de las ecuaciones [2], [3] y [4], y posteriormente representadas en los diagramas, corresponden para todos los DMD con el número de árboles por hectárea y la altura dominante del grupo de especies de coníferas, respectivamente. Sin embargo, los valores de $d_{\mathrm{g}}$ usados en los ajustes corresponden con los del grupo específico de especies para el que se construyó el diagrama. El ajuste de los parámetros se realizó por el método de máxima verosimilitud con información completa (FIML), mediante el procedimiento MODEL de SAS/ETS (SAS Institute Inc 2004).

Para establecer la línea del autoaclareo, o el límite de máxima densidad en los DMD, se ajustó por regresión quantílica no lineal (Koenker y Bassett 1978) una ecuación potencial que relaciona el número de árboles por hectárea con los valores de altura dominante para el grupo de especies de coníferas con los dos niveles de productividad:

$$
N=a_{1} H_{0}^{a_{2}}
$$

Donde, $a_{1}$ y $a_{2}=$ parámetros a estimar mediante la minimización de la función [6]:

$$
S=\sum_{N_{i} \geq \hat{N}_{i}} \tau\left|N_{i}-\hat{N}_{i}\right|+\sum_{N_{i}<\hat{N}_{i}}(1-\tau)\left|N_{i}-\hat{N}_{i}\right|
$$

El valor del cuantil $(\tau)$ utilizado en el ajuste fue el del $95 \%$, es decir, el límite de autoclareo solo es superado por el $5 \%$ de las parcelas. La estimación de los parámetros de la ecuación [5] fue obtenida mediante el uso de la función "nlrq" del módulo "quantreg" (Koenker, 2015) del programa R (R Core Development Team 2014).

El análisis de la capacidad de ajuste de los modelos se basó en las gráficas de los residuos y en los valores de dos estadísticos: el coeficiente de determinación $\left(R^{2}\right)$ y la raíz del error medio cuadrático ( $R M S E)$, cuyas expresiones matemáticas son las siguientes:

$$
\begin{aligned}
& R^{2}=1-\frac{\sum_{i=1}^{n}\left(y_{i}-\hat{y}_{i}\right)^{2}}{\sum_{i=1}^{n}\left(y_{i}-\bar{y}\right)^{2}} \\
& R M S E=\frac{\sqrt{\sum_{i=1}^{n}\left(y_{i}-\hat{y}_{i}\right)^{2}}}{n-p}
\end{aligned}
$$

Donde, $y_{i}, \hat{y}_{i}$ e $\bar{y}=$ valores observado, predicho y medio de la variable dependiente, respectivamente; $n=$ número de datos empleados en el ajuste y $p=$ número de parámetros de la ecuación ajustada.

\section{RESULTADOS}

Ajuste de las relaciones alométricas. Todos los parámetros de las ecuaciones que definen los DMD (cuadro 2) resultaron ser significativos con un $95 \%$ de nivel de significancia. El análisis de los residuales reveló que las ecuaciones de regresión no presentaron tendencias anómalas que hicieran suponer un incumplimiento de las hipótesis de partida de normalidad, homogeneidad de varianza e independencia de los errores.

Los mejores ajustes se obtuvieron en las ecuaciones que estiman el volumen del rodal (ecuación [3]); logrando explicar más del $83 \%$ de la variabilidad observada (cuadro 3 ). Los resultados del ajuste de la ecuación [4] que estima el volumen total del rodal $(V)$ son algo dispares, con un valor del coeficiente de determinación de 0,81 para el nivel de productividad alto y de 0,68 para el nivel medio. Cuando se agrupan los dos niveles de productividad, el valor del coeficiente de determinación se sitúa en un valor intermedio, explicando algo más del $78 \%$ de la variabilidad del volumen total. Con respecto a la ecuación que estima el diámetro medio cuadrático (ecuación [2]), se obtuvieron valores del coeficiente de determinación ajustado que oscilaron de 0,50 a 0,55 para el nivel de productividad alto, y de 0,49 a 0,62 para el nivel medio; por lo que de nuevo se observa algo más de variabilidad en este último nivel de productividad. En general, las especies de coníferas muestran mejores estadísticos de bondad de ajuste que las latifoliadas; lo cual se debe a que el manejo practicado en estos bosques se dirige principalmente a estas especies por ser consideradas de mayor interés económico.

En cuanto a los valores del estimador del error estándar de las ecuaciones (RMSE), en la ecuación [2] los valores oscilaron de 2,46 a 3,08 cm para el grupo coníferas, y de 4,55 a 5,16, para el grupo latifoliadas, correspondiéndose en ambos casos el valor más bajo para nivel de productividad medio; debido a que los valores de $d_{\mathrm{g}}$ en esos rodales es más bajo. Los valores obtenidos de este estadístico en la ecuación [3] presentan importantes diferencias entre grupos de especies y niveles de productividad con valores 
Cuadro 2. Estimación de parámetros en el ajuste simultáneo de las ecuaciones [2], [3] y [4] para los grupos de especies y los niveles de productividad estudiados.

Estimation of parameters in the simultaneous adjustment of equations [2], [3] and [4] for groups of species and productivity studied levels.

\begin{tabular}{|c|c|c|c|c|c|c|c|c|c|c|c|}
\hline \multirow{2}{*}{ Nivel de productividad } & \multicolumn{11}{|c|}{ Parámetros estimados de las ecuaciones [2], [3] y [4] } \\
\hline & $\boldsymbol{\beta}_{1}$ & $\boldsymbol{\beta}_{2}$ & $\boldsymbol{\beta}_{3}$ & $\boldsymbol{\beta}_{4}$ & $\boldsymbol{\beta}_{5}$ & $\boldsymbol{\beta}_{6}$ & $\boldsymbol{\beta}_{7}$ & $\boldsymbol{\beta}_{8}$ & $\boldsymbol{\beta}_{9}$ & $\boldsymbol{\beta}_{10}$ & $\boldsymbol{\beta}_{11}$ \\
\hline \multicolumn{12}{|c|}{ Coníferas } \\
\hline Alto & 36,72 & $-0,21$ & 0,31 & 0,0007 & 1,61 & 0,80 & 0,89 & - & - & - & - \\
\hline Medio & 36,56 & $-0,24$ & 0,34 & 0,0021 & 1,37 & 0,70 & 0,95 & - & - & - & - \\
\hline \multicolumn{12}{|c|}{ Latifoliadas } \\
\hline Alto & 14,94 & $-0,13$ & 0,57 & 0,0011 & 1,45 & 0,83 & 0,89 & - & - & - & - \\
\hline Medio & 14,71 & $-0,12$ & 0,52 & 0,0020 & 1,26 & 0,79 & 0,95 & - & - & - & - \\
\hline \multicolumn{12}{|c|}{ Total (coníferas + latifoliadas) } \\
\hline Alto & 21,14 & $-0,19$ & 0,42 & 0,0010 & 1,42 & 0,77 & 1,01 & 0,0005 & 1,64 & 0,88 & 0,80 \\
\hline Medio & 45,49 & $-0,28$ & 0,35 & 0,0025 & 1,35 & 0,68 & 0,95 & 0,0011 & 1,54 & 0,80 & 0,77 \\
\hline Alto + Medio & 26,90 & $-0,19$ & 0,37 & 0,0004 & 1,67 & 0,85 & 0,91 & 0,0004 & 1,68 & 0,90 & 0,78 \\
\hline
\end{tabular}

Cuadro 3. Estadísticos de ajuste de regresión no lineal para los grupos de especies y niveles de productividad estudiados.

Adjusting statistics of nonlinear regression for the groups of species and productivity levels studied.

\begin{tabular}{|c|c|c|c|c|c|c|}
\hline \multirow{2}{*}{ Nivel de productividad } & \multicolumn{3}{|c|}{$R^{2}$ (Ecuación) } & \multicolumn{3}{|c|}{$R M S E$ (Ecuación) } \\
\hline & [2] $d_{g}$ & {$[3] \mathrm{V}$} & [4] $V_{t}$ & [2] $d_{g}(\mathrm{~cm})$ & [3] $V\left(\mathrm{~m}^{3} \mathrm{ha}^{-1}\right)$ & [4] $V_{t}\left(\mathrm{~m}^{3} \mathrm{ha}^{-1}\right)$ \\
\hline \multicolumn{7}{|c|}{ Coníferas } \\
\hline Alto & 0,52 & 0,83 & - & 3,08 & 29,45 & - \\
\hline Medio & 0,62 & 0,84 & - & 2,46 & 16,25 & - \\
\hline \multicolumn{7}{|c|}{ Latifoliadas } \\
\hline Alto & 0,50 & 0,87 & - & 5,16 & 18,10 & - \\
\hline Medio & 0,49 & 0,83 & - & 4,55 & 9,12 & - \\
\hline \multicolumn{7}{|c|}{ Total (coníferas + latifoliadas) } \\
\hline Alto & 0,55 & - & 0,81 & 2,67 & - & 23,42 \\
\hline Medio & 0,59 & - & 0,68 & 2,57 & - & 25,36 \\
\hline Alto + Medio & 0,51 & - & 0,78 & 2,99 & - & 42,45 \\
\hline
\end{tabular}

de $29,45 \mathrm{~m}^{3} \mathrm{ha}^{-1}$ y $16,25 \mathrm{~m}^{3} \mathrm{ha}^{-1}$ para el grupo coníferas y nivel de productividad alto y medio, respectivamente, mientras que para estos mismos niveles en el grupo latifoliadas los valores fueron de $18,1 \mathrm{~m}^{3} \mathrm{ha}^{-1}$ y $9,12 \mathrm{~m}^{3} \mathrm{ha}^{-1}$, respectivamente. Los valores de este estadístico tienen un comportamiento lógico, son más bajos en la descripción de la relaciones alométricas en las latifoliadas y niveles de productividad medios, como una consecuencia de que los volúmenes de latifoliadas son más bajos que los de las especies de coníferas y que, a su vez, a igualdad del resto de variables dasométricas, los volúmenes del nivel de productividad alto son mayores que los del nivel de productividad medio.

Diagramas de manejo de la densidad. En la figura 2 se muestra, a modo de ejemplo, el uso del DMD para las especies de coníferas localizadas en aquellos rodales con la mayor productividad del predio, cuyo objetivo de gestión será la producción de madera para asierre y, el resto de los DMD desarrollados se muestran en las figuras 3 a 8 . 


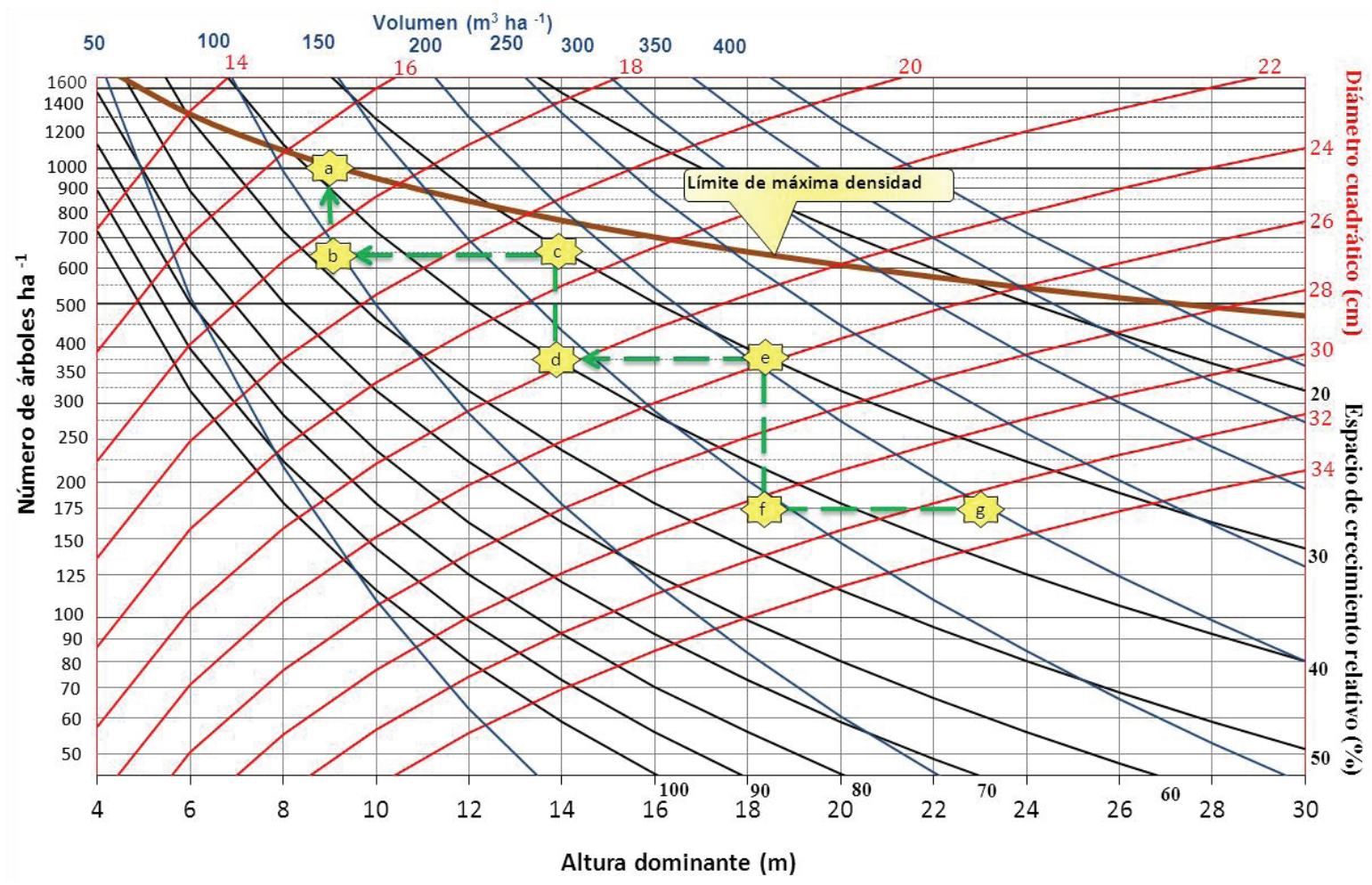

Figura 2. Ejemplo de uso de los DMD para el grupo de especies de coníferas para el nivel de productividad alto.

Example use of DMD for coniferous species group to the highest level of productivity.

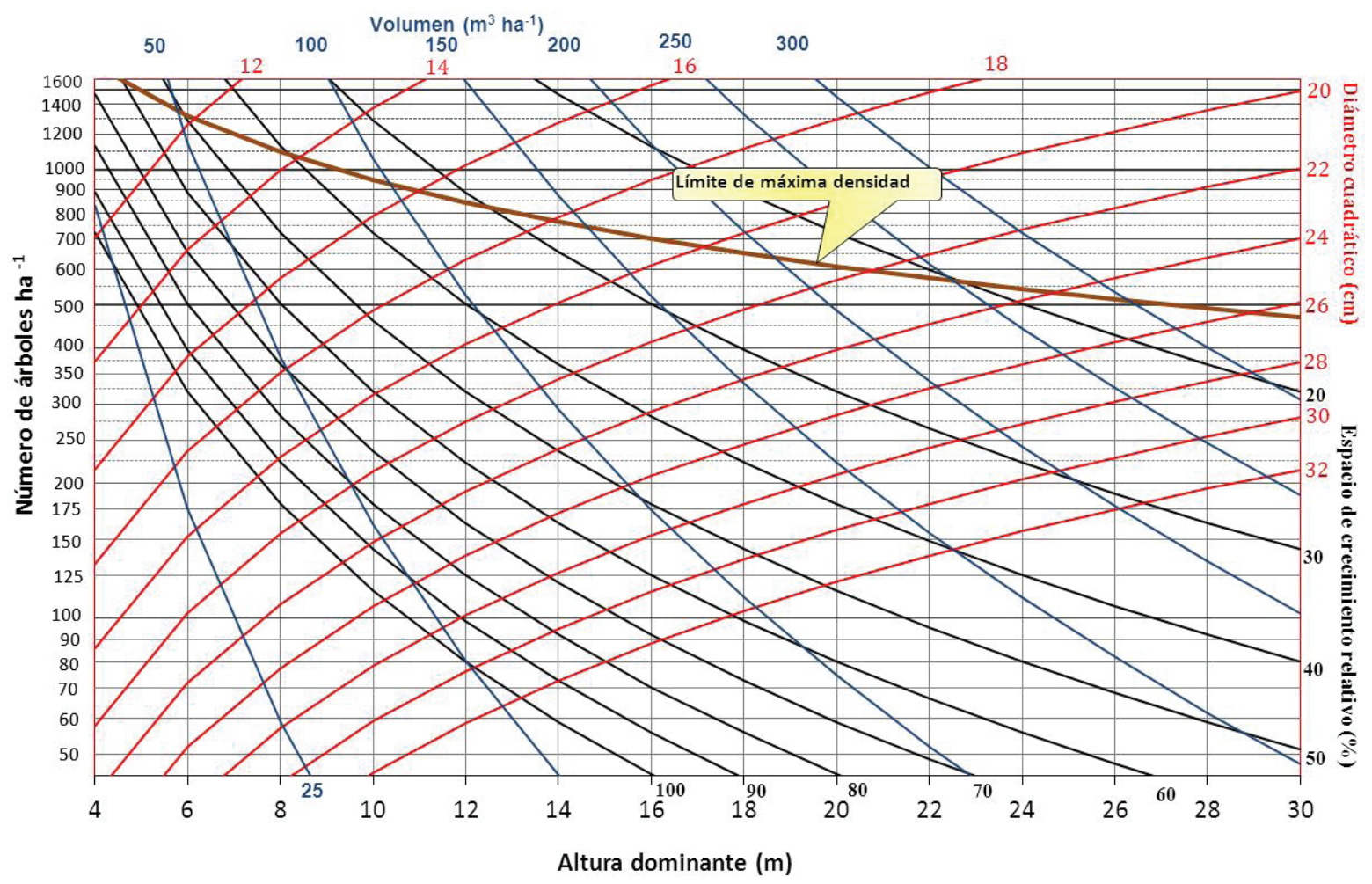

Figura 3. DMD para el grupo de especies de coníferas para el nivel de productividad medio.

DMD for the group of coniferous species for the average level of productivity. 


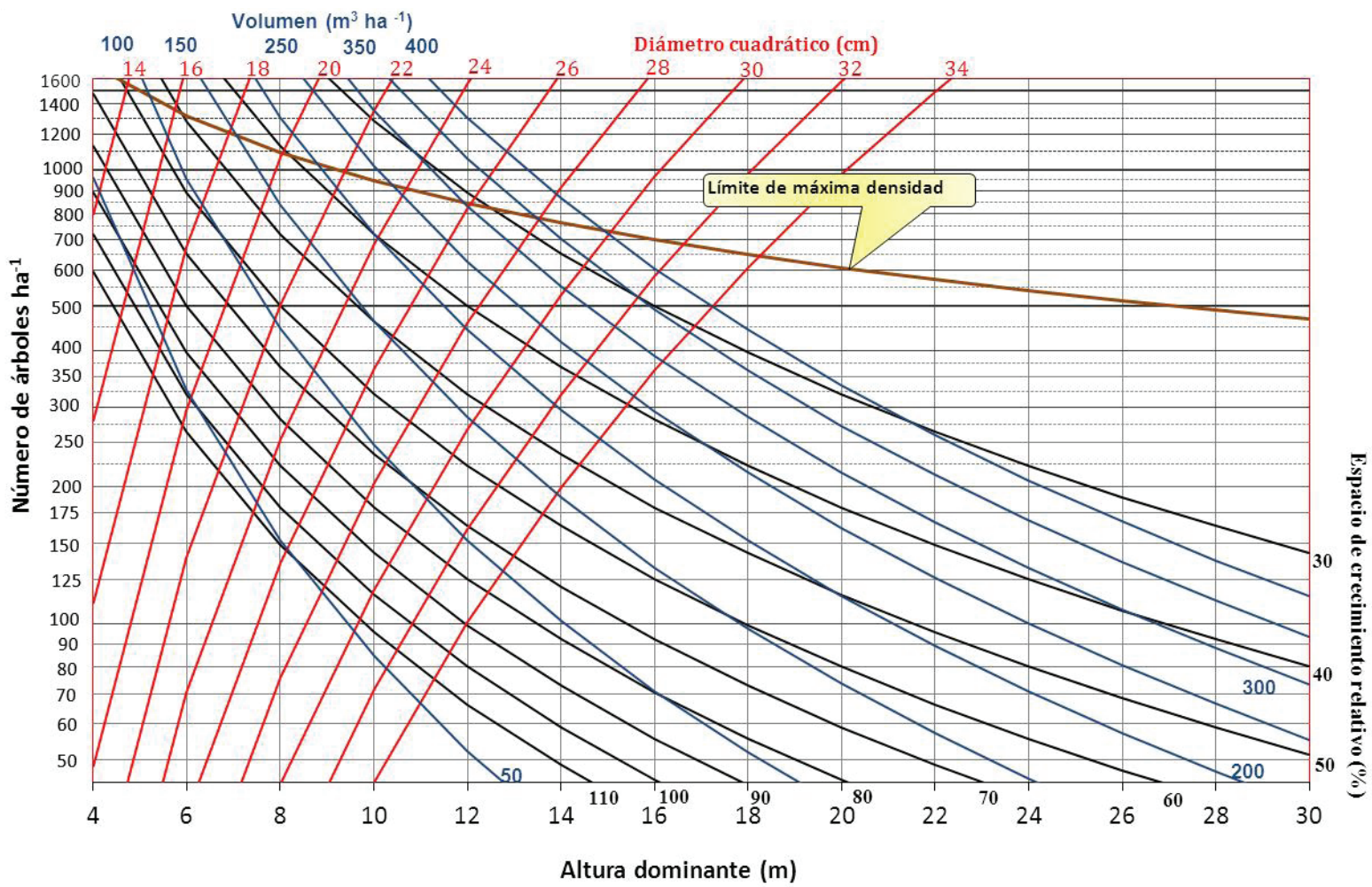

Figura 4. DMD para el grupo de especies de latifoliadas para el nivel de productividad alto.

DMD for the group of hardwood species for the high level of productivity.

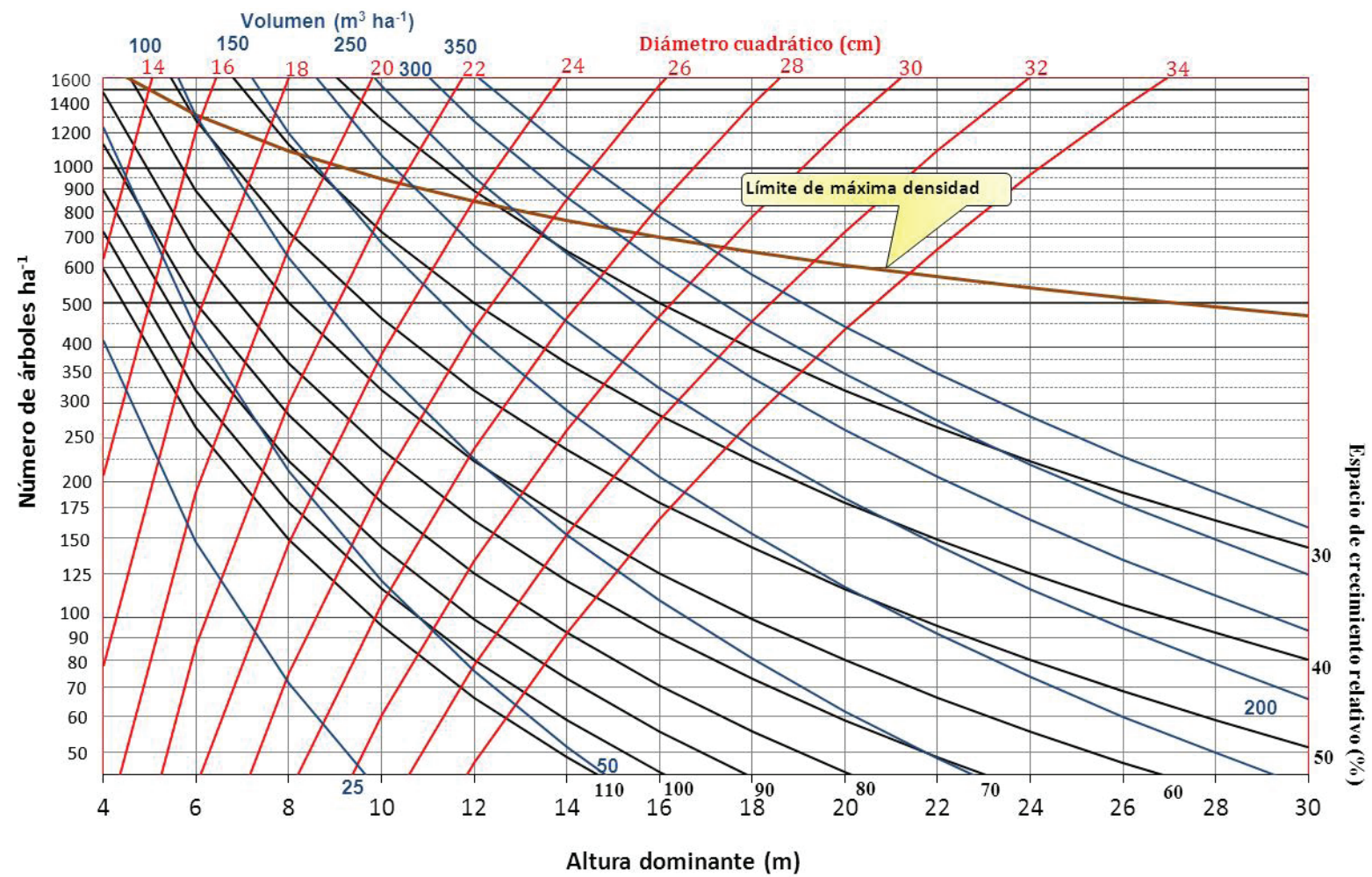

Figura 5. DMD para el grupo de especies de latifoliadas para el nivel de productividad medio.

DMD for the group of hardwood species for the average level of productivity. 


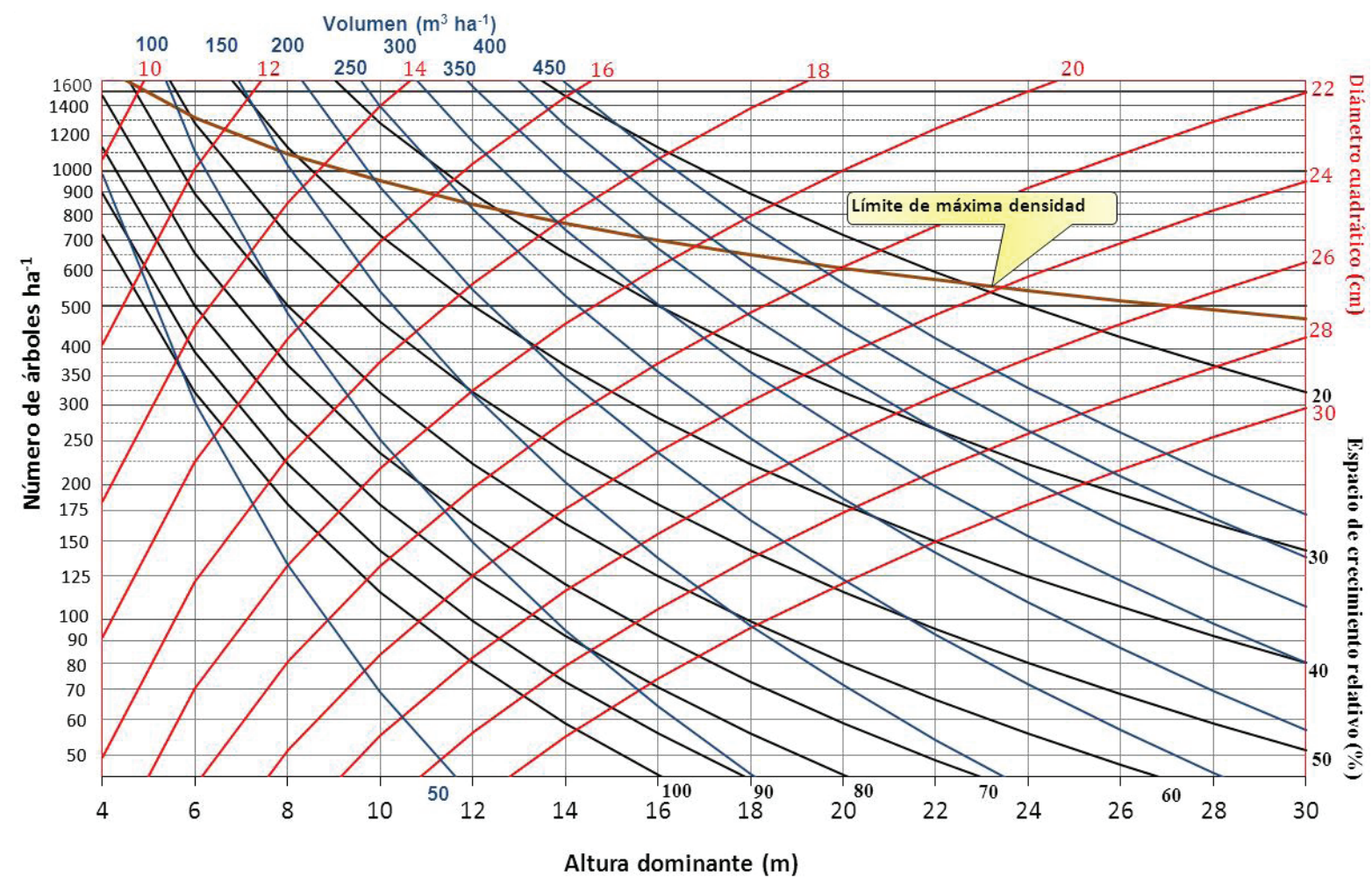

Figura 6. DMD para rodales mixtos para el nivel de productividad alto.

DMD for mixed stands and for the high level of productivity.

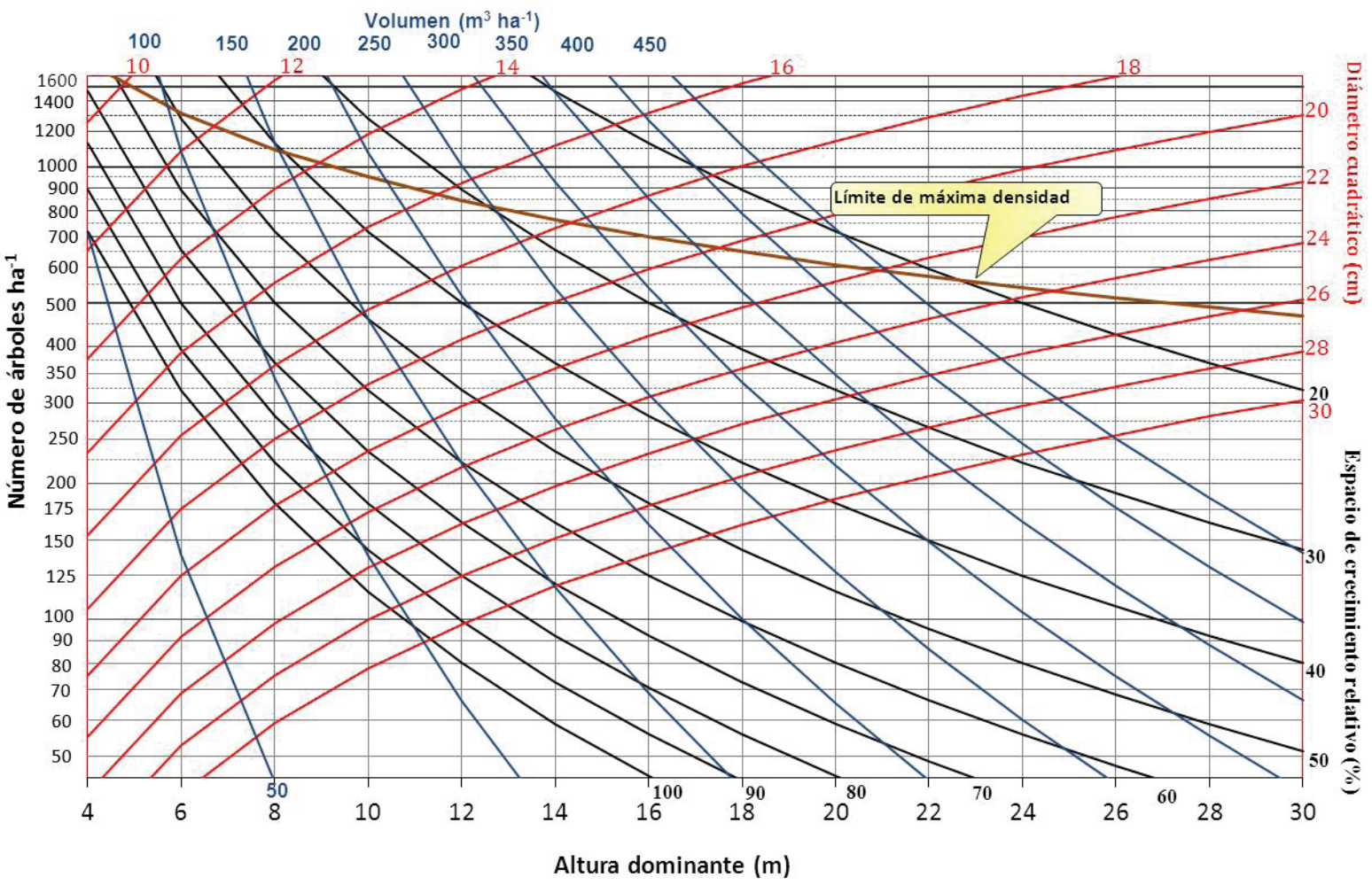

Figura 7. DMD para rodales mixtos para el nivel de productividad medio.

DMD for mixed stands and the average level of productivity. 


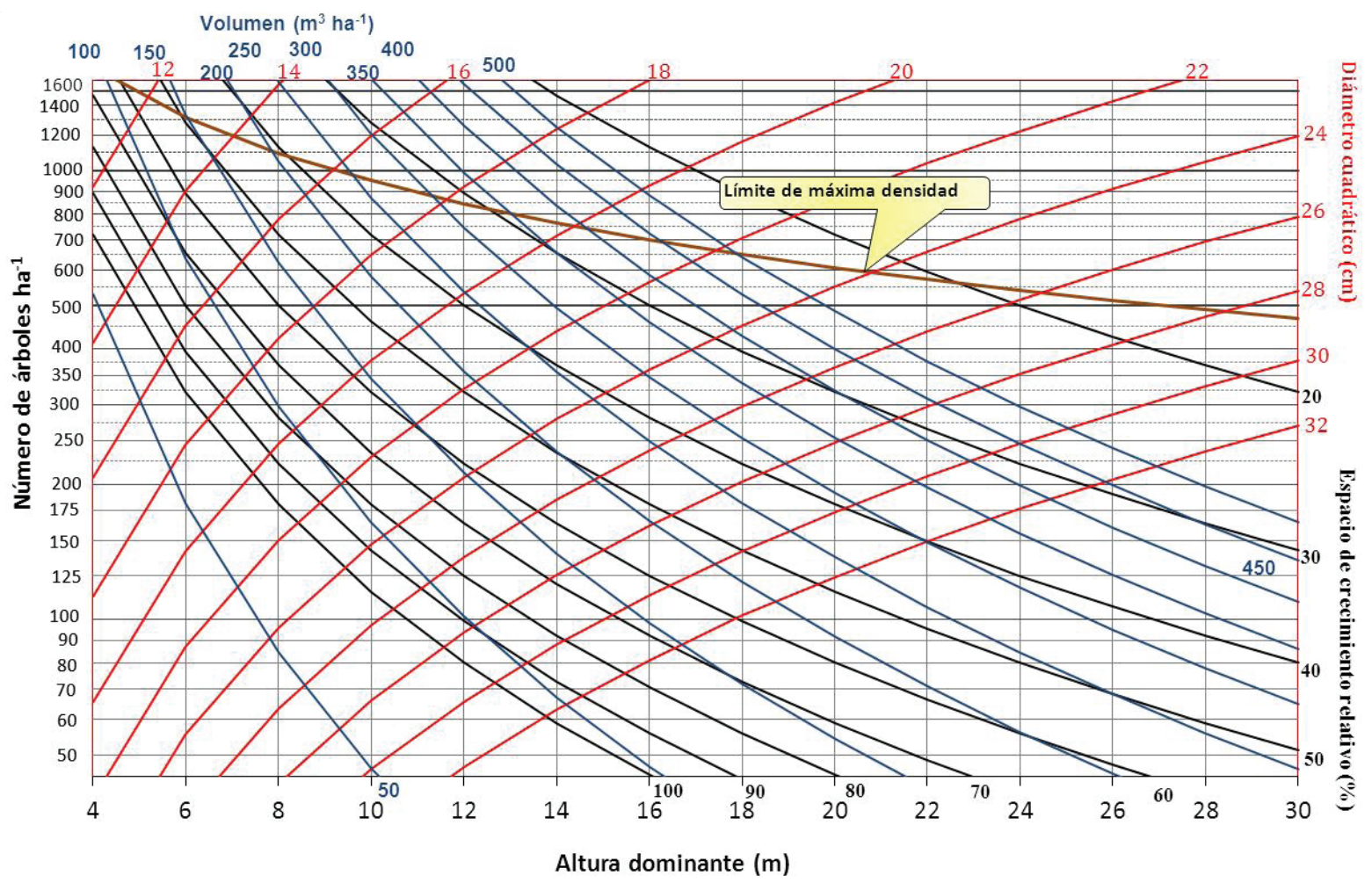

Figura 8. DMD para rodales mixtos para los dos niveles de productividad.

DMD for mixed stands and both productivity levels.

Límite máximo del nivel de densidad. Los estimadores de los parámetros de la ecuación que determina el límite máximo de densidad o línea de autoclareo (ecuación [5]) obtenidos en el ajuste de regresión cuantílica (95\%) fueron 4.146,3 y -0,6403 para el término independiente y el exponente de la altura dominante, respectivamente. Ambos estimadores fueron significativos al $95 \%$.

A modo orientativo, en el cuadro 4 , se muestran los valores promedio de altura dominante y diámetro medio cuadrático a fijar como objetivo final en función del grupo de especies considerado y del nivel de productividad del rodal. También se muestran los límites del índice de espacio de crecimiento relativo que no deberían sobrepasarse. Todos estos valores indicativos se basan en los criterios establecidos en el Programa de Manejo Forestal del área de estudio (UCODEFO 2006) y considerando los índice de sitio de 15 y 12 metros de altura dominante a la edad de referencia de 60 años para los niveles de productividad alto y medio, respectivamente.
Cuadro 4. Valores promedio de diámetro medio cuadrático e índice de espaciamiento relativo de los DMD de los grupos de especies para una altura dominante objetivo de producción en rodales mixtos e irregulares del ejido San Diego de Tezains.

Average values of quadratic mean diameter and relative spacing index of the DMD of the species groups for an objective dominant height of production in mixed and uneven-aged stands in the Ejido San Diego Tezains.

\begin{tabular}{|c|c|c|c|c|}
\hline $\begin{array}{l}\text { Grupo de } \\
\text { especies }\end{array}$ & $\begin{array}{c}H_{0} \text { objetivo } \\
(\mathrm{m})\end{array}$ & $\begin{array}{c}\text { Nivel de } \\
\text { productividad }\end{array}$ & $\begin{array}{c}d_{g} \\
(\mathrm{~cm})\end{array}$ & $\begin{array}{l}R S \\
(\%)\end{array}$ \\
\hline \multirow{2}{*}{ Coníferas } & \multirow{2}{*}{25} & Alto & 28,0 & 21 \\
\hline & & Medio & 25,0 & 19 \\
\hline \multirow{2}{*}{ Latifoliadas } & \multirow{2}{*}{15} & Alto & 33,0 & 40 \\
\hline & & Medio & 30,0 & 37 \\
\hline \multirow{3}{*}{ Total } & \multirow{3}{*}{20} & Alto & 22,5 & 22 \\
\hline & & Medio & 22,0 & 23 \\
\hline & & Alto + Medio & 25,0 & 25 \\
\hline
\end{tabular}




\section{DISCUSIÓN}

Los valores del coeficiente de determinación obtenidos para las diferentes ecuaciones ajustadas han sido aceptables, aunque están, en general, por debajo de los valores obtenidos por otros autores. Por ejemplo, Barrio-Anta y Álvarez (2005) obtuvieron coeficientes de determinación de 0,86 y 0,98 para las ecuaciones [2] y [3], respectivamente en rodales de Quercus robur L. en Galicia; Castedo-Dorado et al. (2009) obtuvieron valores de 0.92 y 0.99 para las mismas ecuaciones en rodales de Pinus radiata $\mathrm{D}$. Don en el noroeste de España; y Tewari y Álvarez-González (2014) valores de 0.74 y 0.98 , respectivamente, en rodales de Tectona grandis L. en la India. En todo caso, hay que tener en cuenta que todos estos trabajos fueron realizados en plantaciones o masas regulares con pautas de crecimiento mucho más homogéneas que los bosques mixtos e irregulares analizados en este estudio.

Para el uso de los DMD desarrollados en este trabajo existen dos factores que resultan de importancia a considerar en la definición de un programa de aclareos: (i) el estatus del rodal que marca el objetivo final; y (ii) la definición de los límites superior e inferior de la densidad del rodal. El estatus del rodal puede ser establecido en términos de dos cualesquiera de las variables que figuran en el diagrama: número de árboles por hectárea, altura dominante, índice de espacio de crecimiento relativo, diámetro medio cuadrático y volumen (e.g., Valbuena et al. 2008). La definición del límite superior de los DMD se fundamenta en evitar que los bosques naturales crucen la línea del autoaclareo; es decir, que no existan pérdidas por mortalidad natural. Mientras que el límite inferior estará determinado en función de los objetivos del manejo de las especies presentes en el rodal tratando de mantener siempre ocupado el espacio de crecimiento, considerando un nivel superior del cierre de copas.

Uso de los diagramas para el manejo de la densidad $(D M D)$. En este trabajo se desarrollaron siete DMD (figuras 2 a 8), los cuales muestran las isolíneas del índice de espacio de crecimiento relativo, diámetro medio cuadrático y volumen del rodal en una gráfica de dos dimensiones. En el eje de las abscisas se encuentra la altura dominante del rodal (obtenida del grupo de las coníferas) y, en el eje de las ordenadas, la densidad del rodal expresada como el número de árboles por hectárea del grupo para el cual se construyó el diagrama.

En el caso del DMD del grupo de especies coníferas en su nivel de productividad alto (figura 2), en el eje que corresponde a la altura dominante, el rango establecido es de 4 a $30 \mathrm{~m}$, mientras que en el eje que corresponde a la densidad del rodal se muestra un intervalo de 50 a 1.600 árboles por hectárea en una escala logarítmica. Estos valores coinciden con los observados en las bases de datos utilizadas en los ajustes.

El índice de espacio de crecimiento relativo en los bosques seminaturales permitirá definir el momento adecuado para realizar los aclareos y el intervalo de tiempo entre ellos en función del objetivo que se pretenda alcanzar. Sus valores están representados por las isolíneas que definen el grado de densidad que existe en el rodal en función de la altura dominante (que está estrechamente relacionada con la edad e índice de sitio del rodal). Para los DMD, aquí desarrollados, los valores del índice $R S$ varían entre el 20 y $110 \%$ en los grupos de especies y niveles de productividad estudiados. En tanto que para las isolíneas del diámetro medio cuadrático sus valores o rangos oscilan de 10 a 34 $\mathrm{cm}$, aunque con algunas pequeñas diferencias dependiendo del grupo de especies y del nivel de productividad representado. Por su parte, las isolíneas que determinan el volumen del rodal, presentan valores en un rango de 25 a $450 \mathrm{~m}^{3} \mathrm{ha}^{-1}$. Todos estos límites se han establecido teniendo en cuenta los valores observados en los datos de campo empleadas para realizar los ajustes.

En la figura 2 se muestra un ejemplo de uso de los diagramas de manejo de la densidad. En primer lugar, se ha fijado como objetivo final la producción de madera para asierre o chapa bajo la definición de una altura dominante $\left(H_{0}\right)$ de 23 metros y un diámetro medio cuadrático $\left(d_{\mathrm{g}}\right)$ de $33 \mathrm{~cm}$. Estas dos condiciones definen el punto " $g$ " del gráfico y, a su vez, para este punto resulta una densidad de 175 árboles ha ${ }^{-1}$, un índice de espacio de crecimiento relativo de aproximadamente $35 \%$ y existencias maderables muy cercanas a los $200 \mathrm{~m}^{3} \mathrm{ha}^{-1}$. Del mismo modo, se fija como límite superior de la densidad el que marca un valor del índice de espacio de crecimiento relativo de un $30 \%$, siempre y cuando se esté por debajo de la línea de autoaclareo. Teniendo en cuenta lo establecido en el Programa de Manejo Forestal de este ejido (UCODEFO 2006), para lograr esta meta es necesario realizar cortas con ciclos de 15 años. La corta previa a alcanzar el objetivo final se realiza 15 años antes (un ciclo), pero puesto que en los diagramas no figura el tiempo como variable, se estima la altura dominante del rodal en 15 años antes de alcanzar el valor de 23 metros; empleando la ecuación que define la altura dominante del rodal para estos bosques naturales y que se muestra en la expresión de la ecuación [9] (UCODEFO 2006):

$$
H_{0}=2,199 \cdot S^{0,936} \cdot[1-\exp (-0,0304 \cdot t)]^{4,784 \cdot S^{-0,361}}[9]
$$

Donde, $S=$ índice de sitio del rodal; $t=$ edad en años.

En consecuencia, considerando una calidad de estación para el nivel de productividad de 16 metros a una edad de referencia de 60 años, se obtiene una altura dominante de 18,5 metros; un ciclo antes de alcanzar el objetivo final. Por lo tanto, la situación tras la última intervención se obtiene trazando una horizontal desde el punto " $g$ " hasta una altura dominante de 18,5 metros, fijando entonces el punto " $f$ ". Al trazar una línea horizontal, se asume que no hay mortalidad ni tampoco se producen incorporaciones de nuevos árboles en ese período tras la intervención. Si 
se considera que esa situación no es realista, se podrían incluir en el modelo unas ecuaciones de mortalidad y de incorporación; con lo que la línea tendría una cierta pendiente que podría ser negativa o positiva en función del balance de pérdidas y ganancias de árboles. La situación antes de la corta se obtiene al trazar una línea vertical hasta alcanzar el límite superior de densidad fijado, marcando el punto " $e$ ". Al trazar una línea vertical se asume que la corta realizada no afecta a la altura dominante del rodal, en caso contrario, la recta tendría una cierta pendiente negativa como consecuencia de la reducción de la altura dominante. A partir de ese punto, de nuevo se estima la altura dominante del rodal 15 años antes (dos ciclos para llegar al punto objetivo), obteniendo un valor de 13,8 metros, y se continúa desplazando, como una escalera, la línea que define la alternativa silvícola adecuada para alcanzar el objetivo final buscado. De este modo, si se fijan tres intervenciones (en tres ciclos), el punto de partida sería el " $a$ "; definido por una altura dominante de $9,2 \mathrm{~m}$, una densidad de 1000 árboles ha ${ }^{-1}$, un índice de espacio de crecimiento relativo del $37 \%$, lo que supone una distancia media entre árboles de $3,4 \mathrm{~m}$, un $d_{\mathrm{g}}$ de $17 \mathrm{~cm}$ y un volumen muy cercano a $120 \mathrm{~m}^{3} \mathrm{ha}^{-1}$. Cada punto marcado en el diagrama permite estimar los valores de densidad, altura dominante, diámetro medio cuadrático, índice de espacio de crecimiento relativo y volumen, por lo que se pueden estimar, por ejemplo, los volúmenes obtenidos en las intervenciones como diferencia entre los valores antes y después de la misma (pares de puntos “ $a-b$ ", " $c-d$ " y "e- $f$ " de la figura 2), aunque estos volúmenes son valores promedio y pueden variar notablemente en función de las clases diamétricas afectadas en las cortas.

\section{CONCLUSIONES}

Los resultados obtenidos en este trabajo han ratificado la hipótesis de que los DMD pueden modelizar la evolución de masas mixtas e irregulares con similar exactitud a cuando se emplean en plantaciones forestales. Los DMD desarrollados se pueden adaptar a un amplio rango de curvas o líneas de referencia en la planificación de las cortas para lograr objetivos tales como: producción maderable con la calidad que demanda el mercado actual, protección de incendios y plagas forestales, mantener los servicios ambientales que presta el bosque (conservación de la biodiversidad, hábitat para la vida silvestre, producción de agua y recreación, etc.).

La altura dominante del rodal es la principal variable a considerar en la definición del período y frecuencia de las intervenciones, que a su vez dependerá de las tasas de crecimiento como un indicador de la productividad y de la composición de especies y edad del rodal.

Los DMD desarrollados permiten realizar una adecuada planificación y seguimiento de las intervenciones silvícolas, maximizando la utilización de la productividad del sitio mediante un control adecuado de los niveles de competencia del rodal. La línea de referencia que definen la densidad del auto-aclareo fue establecida considerando el grupo de especies de mayor dominancia (coníferas).

Finalmente, destacar que la posibilidad de utilizar los DMD para los bosques del noroeste del estado de Durango es una ventaja clara con respecto a la construcción y aplicación de modelos dinámicos de rodal que requieren mayor cantidad de datos y variables involucradas.

\section{REFERENCIAS}

Aguirre CO, G Vega, J Jiménez. 1994. Guía de densidad para Pinus pseudostrobus Lindl. Nuevo León. In Memorias del Simposio y II Reunión Nacional de Silvicultura y Manejo de Recursos Forestales. México DF, México. 43 p.

Assmann E. 1961. Waldertragskunde. München, Alemania. BLV Verlagsgesellschaft. 490 p.

Barrio-Anta M, JG Álvarez-González. 2005. Development of a stand density management diagram for even-aged pedunculate oak stands and its use in designing thinning schedules. Forestry 78 (3): 209-216.

Castedo-Dorado F, F Crecente-Campo, P Álvarez-Álvarez, M Barrio-Anta. 2009. Development of a stand density management diagram for radiata pine stands including assessment of stand stability. Forestry 82 (1): 1-16.

Dean TJ, VC Baldwin. 1993. Using a density management diagram to develop thinning schedules for loblolly pine plantations. New Orleans, USA. USDA Forest Service, Southern Forest Experimental Station. 10 p. (Research Paper SO 275).

Dean TJ, VC Baldwin. 1996. Crown management and stand density. In Carter MC ed. Growing Trees in a Greener World: Industrial Forestry in the 21st Century. 35th LSU Forestry Symposium. Baton Rouge LA, USA. Louisiana State University Agricultural Center, Louisiana Agricultural Experiment Station. p. 148-159.

Drew TJ, JW Flewelling. 1979. Stand density management: an alternative approach and its application to Douglas-fir plantations. Forestry Science 25: 518-532.

Duplat P, P Champagne, F Chollet, C Ginisty, P Jarret, M Nouveau, F Reteau, E Sevrin. 1996. Sylviculture du chêne pédonculé. ONF Bulletin Technique 31: 15-19.

Gadow KV, GY Hui. 1999. Modelling forest development. Dordrecht, The Netherlands. Kluwer Academic Publisher. 213 p.

García ME. 1981. Modificaciones al sistema de clasificación climática de Köppen. $4^{\mathrm{a}}$ ed. México DF, México. Instituto de Geografía, Universidad Nacional Autónoma de México. $90 \mathrm{p}$.

Koenker R. 2015. quantreg: Quantile Regression. R package version 5.11. http://CRAN.R-project.org/package=quantreg,

Koenker R, G Bassett. G 1978. Regression quantiles. Econometrica 46(1): 33-50.

Long JN. 1985. A practical approach to density management. The Forestry Chronicle 23: 23-26.

López-Sánchez CA, R Rodríguez-Soalleiro. 2009. A Density Management Diagram Including Stand Stability and Crown Fire Risk for Pseudotsuga Menziesii (Mirb.) Franco in Spain. Mountain Research and Development 29(2): 169-176.

Márquez MA, R Álvarez. 1995. Construcción de una guía de densidad para Pinus Cooperi var. ornelasi con base en la clase de copa en Durango, México. Madera y Bosques 1(2): 
23-36.

Newton PF. 1997. Stand density management diagrams: review of their development and utility instand-level management planning. Forest Ecology and Management 98: 251-265.

Newton PF, GF Weetman. 1994. Stand density management diagram for managed black spruce stands. The Forestry Chronicle 70: 65-74.

Pérez-Cruzado C, A Merino, R Rodríguez- Soalleiro. 2011. A management tool for estimating bioenergy production and carbon sequestration in Eucalyptus globulus and Eucalyptus nitens grown as short rotation woody crops in northwest Spain. Biomass and Bioenergy 35: 2839- 2851.

R Core Team. 2014. R: A Language and Environment for Statistical Computing. R Foundation for Statistical Computing, Vienna, Austria. http://www.R-project.org/.

Reineke LH. 1933. Perfecting a stand density index for evenaged forest. Journal of Agricultural Research 46: 627-638.

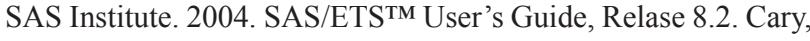
NC, USA.

SRNyMA (Secretaria de Recursos Naturales y Medio Ambiente del Gobierno del Estado, MX). 2006. Programa Estratégico Forestal 2030. Victoria de Durango, Durango, México. Secretaría de Recursos Naturales y Medio Ambiente del Estado de Durango. 242 p.

Schütz JP. 1990. Sylviculture. I. Princies d'education des fôrets. Laussanne, France. Presses Polytechniques et Universita- ries Romandes. 243 p.

Tadaki Y. 1963. The pre-estimating of stem yield based on the competition density effect. Ringyo Shikenjo Kenkyu Hokoku 155: 1-19.

Tewari VP, JG Álvarez-González. 2014. Development of a Stand Density Management Diagram for Teak Forests in Southern India. Journal of Forest and Environmental Sciences 30(3): 259-266.

UCODEFO (Unidad de Conservación y Desarrollo Forestal, MX). 2006. Programa de Manejo Forestal 2006-2020. Santiago Papasquiaro, Durango, México. Unidad de Conservación y Desarrollo Forestal Nuevo San Diego de Tenzaenz.

Valbuena P, C del Peso, F Bravo. 2008. Stand Density Management Diagrams for two Mediterranean pine species in Eastern Spain. Investigación Agraria: Sistemas y Recursos Forestales 17(2): 97-10.

Wehenkel C, Corral-Rivas JJ, Hernández-Díaz JC, Gadow KV. 2011. Estimating balanced structure áreas in multi-species forests on the Sierra Madre Occidental, Mexico. Annals of Forest Science 68: 385-394.

Wilson FG. 1946. Numerical expression of stocking in terms of height. Journal of Forestry 44: 758-761.

Yoda K, H Kira, H Ogawa, K Hozumi. 1963. Self-thinning in overcrowded pure stands under cultivated and natural conditions. Journal of Biology (Osaka City University) 14: 107-129. 
